\title{
Early Developmental Destruction of Terminals in the Striatal Target Induces Apoptosis in Dopamine Neurons of the Substantia Nigra
}

\author{
Maria J. Marti, Christopher J. James, Tinmarlar F. Oo, William J. Kelly, and Robert E. Burke \\ Department of Neurology, College of Physicians and Surgeons, Columbia University, New York, New York 10032
}

Many developing neural systems with peripheral projections depend on their target for trophic support during a critical period of natural cell death. Much less is known about central systems. That dopaminergic neurons of the substantia nigra may depend on their target, the striatum, during development is suggested by the presence of a natural apoptotic cell death event in these neurons that can be augmented by an early developmental axon-sparing striatal injury. To further assess the target dependence of these neurons, we have used the selective neurotoxin 6-hydroxydopamine to lesion their terminals within the striatum during development, while sparing intrinsic striatal target neurons. This lesion results in an induction of apoptotic cell death in phenotypically defined dopaminergic neurons that appears on the third postlesion day and

In many developing neural systems, there have been clear demonstrations of dependence on target structures, such that an experimental decrease in target size results in an augmented level of developmental cell death, which in turn leads to a diminished number of neurons surviving into maturity (Cowan et al., 1984; Clarke, 1985; Oppenheim, 1991). In these paradigms, there is much evidence that the target provides limiting quantities of neurotrophic factors that are transported retrogradely (Barde, 1989) and regulate the magnitude of developmental cell death. Although these concepts have received extensive experimental support from studies of systems with peripheral targets, much less is known about target dependence in developing central neurons with central targets. The evidence that does exist, as from studies of chimeras of cerebellar mutants (Wetts and Herrup, 1983), would suggest that target dependence exists in the context of central development as well. It is unknown whether these concepts of classic neurotrophic theory apply to developing mesencephalic dopaminergic systems. These issues are important to examine, because these systems play a major role in the pathophysiology of many common and disabling human neurological and psychiatric diseases, including Parkinson's disease, schizophrenia, and addictive behaviors (Giros et al., 1996).

We have shown that early developmental excitotoxic injury to the target striatum results in a diminished adult number of dopaminergic neurons in the substantia nigra (SN) (Burke et al., 1992; Macaya and Burke, 1992). This decrease occurs

Received Dec. 19, 1996; accepted Dec. 31, 1996.

This work was supported by National Institutes of Health Grant NS26836, the Parkinson's Disease Foundation, and the Hospital Clinic i Provincial. We are grateful to Ms. Pat White for diligent secretarial assistance.

Correspondence should be addressed to Dr. Robert E. Burke, Box 67, Department of Neurology, College of Physicians and Surgeons, Columbia University, 710 West 168th Street, New York, NY 10032.

Copyright (C) 1997 Society for Neuroscience $\quad 0270-6474 / 97 / 172030-10 \$ 05.00 / 0$ persists until the tenth. This inducibility of cell death is dependent on developmental age: it is most marked before postnatal day (PND) 14. As late as PND42, inducibility is still detectable but much less so. In addition, at day 42 the morphology of cell death changes and becomes nonapoptotic in some cells. We conclude that terminal injury during a critical period of postnatal development, like axon-sparing target injury, induces augmented apoptotic death in mesencephalic dopaminergic neurons. These results suggest that these neurons have a period of target dependence. Regulation of this dependence is likely to influence the mature adult number of dopaminergic neurons.

Key words: apoptosis; programmed cell death; 6-hydroxydopamine; substantia nigra; dopamine; Parkinson's disease

despite the axon-sparing nature of this injury (Schwarcz et al., 1983; Macaya and Burke, 1992) and in the absence of any direct injury to the SN. We have also shown that natural cell death occurs within the SN pars compacta (SNpc) (Janec and Burke, 1993), with the morphology of apoptosis (Kerr et al., 1972), and that this death event is augmented after excitotoxic (Macaya et al., 1994) injury to the striatum during development. These results support the hypothesis that the striatal target may regulate the magnitude of a developmental death event among dopaminergic neurons of the SN. Such a possibility is also supported by many earlier in vitro studies demonstrating the ability of striatal preparations to support the viability of immature dopaminergic neurons (Prochiantz et al., 1979; Hemmendinger et al., 1981; Tomozawa and Appel, 1986).

Nevertheless, the observation of induced apoptotic cell death in SNpc after early striatal excitotoxic injury may have many other possible explanations in addition to the loss of retrograde, targetderived support. After striatal injury, there is a loss of afferent projections to SNpc. There are precedents for afferent regulation of developmental cell death (Linden, 1994), so it is possible that induced cell death in SNpc is attributable to their loss. To differentiate between retrograde and afferent striatal regulation of developmental cell death in dopaminergic neurons, we used the neurotoxin 6-hydroxydopamine (6-OHDA) injected intrastriatally to selectively destroy dopaminergic terminals, without injury to intrinsic striatal neurons or their afferent projections (Ungerstedt, 1971). If developing dopaminergic neurons depend on intact terminal structures for either uptake of a diffusible neurotrophic factor or cell-to-cell contact with target neurons, then their destruction should result in induced developmental cell death. We have therefore studied the effects of developmental intrastriatal injection of 6-OHDA on the magnitude and timing of apoptotic cell death in the SN. 


\section{MATERIALS AND METHODS}

6-OHDA lesions. Female rats 14-16 d pregnant were obtained from Charles River Laboratories (Wilmington, MA). The cage was inspected in the afternoon of each day, and the day of birth was defined as postnatal day (PND) 1. In the first experiment, to identify induced cell death and assess its time course, 50 animals were lesioned at PND7 and killed at varying intervals. Pups were pretreated with $25 \mathrm{mg} / \mathrm{kg}$ desmethylimipramine, anesthetized by inhalation of Metofane (Pittman-Moore), and placed prone on an ice pack. The skull was exposed by a midline incision, and a burr hole was placed $3.0 \mathrm{~mm}$ lateral to the left of bregma on the coronal suture. A 30-gauge cannula was then inserted vertically into the striatum to a depth of $4.0 \mathrm{~mm}$ from the top of the skull. 6-OHDA hydrobromide (Regis) was prepared at $15 \mu \mathrm{g}$ (total weight) $/ 1.0 \mu \mathrm{l}$ in $0.9 \%$ $\mathrm{NaCl} / 0.02 \%$ ascorbic acid, and infused by pump (Carnegie Medicin CMA/100) at a rate of $0.25 \mu \mathrm{l} / \mathrm{min}$. The cannula was withdrawn slowly 2 min after the end of the infusion. Saline $/ 0.02 \%$ ascorbate was injected as a vehicle control. After recovery from anesthesia, pups were returned to the dams until the assigned postlesion day (PLD). In the second experiment, to define the developmental dependence of 6-OHDA effects, 20 animals were studied at PND14, -21, and -28. Animals studied at PND14 were infused using the method described above. At PND21, a cannula was inserted to a depth of $5.0 \mathrm{~mm}$ rather than $4.0 \mathrm{~mm}$; at PND28, the 5.0 $\mathrm{mm}$ depth was used, and animals were anesthetized with pentobarbital 30 $\mathrm{mg} / \mathrm{kg}$ rather than Metofane. Otherwise, infusion conditions were the same. In the third experiment, to assess the time course of 6-OHDA effects at later postnatal ages, 30 animals were studied at PND29 and -42 and killed at PLD5, -7 , and -9 . These animals were infused with 6-OHDA as described above, except that PND42 animals were placed in a stereotaxic frame and injected at coordinates anteroposterior $+0.02 \mathrm{~mm}$, lateral $+0.35 \mathrm{~mm}$, dorsoventral $-0.50 \mathrm{~mm}$ according to the Paxinos-Watson atlas (Paxinos and Watson, 1982), using bregma.

Processing for histological analysis. Animals were anesthetized deeply with either Metofane or pentobarbital and perfused through the ventricle with $0.9 \%$ saline at $4{ }^{\circ} \mathrm{C}$ followed by $4 \%$ paraformaldehyde (PF) in $0.1 \mathrm{M}$

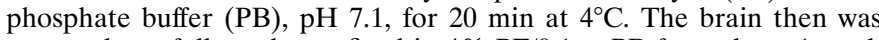
removed carefully and post-fixed in $4 \% \mathrm{PF} / 0.1 \mathrm{M} \mathrm{PB}$ for at least 1 week before it was sectioned. Each brain was cryoprotected in $20 \%$ sucrose in $4 \% \mathrm{PF} / 0.1 \mathrm{M} \mathrm{PB}$ overnight and then frozen rapidly in 2-methylbutane on dry ice. Representative sections $(30 \mu \mathrm{m})$ were taken from each of the major planes encompassing the SN (Paxinos-Watson planes 2.7, 3.2, 3.7, 4.2) (Paxinos and Watson, 1982) and alternately used for silver staining or tyrosine hydroxylase $(\mathrm{TH})$ immunostaining with Nissl counterstain. Representative $30 \mu \mathrm{m}$ sections were also taken from the striatum, from the region within Paxinos-Watson planes 8.7-9.7 (Paxinos and Watson, 1982). These sections were processed for silver impregnation, TH immunostaining, and Nissl staining.

TH immunostaining. Sections $(30 \mu \mathrm{m})$ were incubated overnight at $4^{\circ} \mathrm{C}$ with a mouse monoclonal anti-TH antibody (Boehringer Mannheim, Indianapolis, IN) at 1:10 in PBS/10\% horse serum, followed by incubations with biotinylated horse anti-mouse IgG (Vector Laboratories, Burlingame, CA) at $1: 50$ in $\mathrm{PBS} / 10 \%$ horse serum, and then with avidinbiotinylated horseradish peroxidase complexes (ABC Kit, Vector) at 1:600 at room temperature for $1 \mathrm{hr}$. Sections were then incubated with diaminobenzidine (Aldrich, Milwaukee, WI) $(50 \mathrm{mg} / 100 \mathrm{ml}$ Tris, $\mathrm{pH} 7.6)$ in the presence of $\mathrm{H}_{2} \mathrm{O}_{2}$. Sections were then mounted on gelatin-subbed slides and Nissl-counterstained.

Silver staining. Sections were maintained in serial order and processed free-floating in custom-made plastic grids with nylon mesh bottoms. Sections were collected into cold fixative, washed three times in distilled water, and then immersed in pretreating solution (equal volumes of $9 \%$ $\mathrm{NaOH}$ and $1.2 \% \mathrm{NH}_{4} \mathrm{NO}_{3}$ ) for 5 min twice. They were then immersed in impregnating solution $\left(60 \mathrm{ml}\right.$ of $9 \% \mathrm{NaOH}, 40 \mathrm{ml}$ of $16 \% \mathrm{NH}_{4} \mathrm{NO}_{3}, 0.5$ $\mathrm{ml}$ of $50 \% \mathrm{AgNO}_{3}$ ) for $10 \mathrm{~min}$. Sections were then washed three times in washing solution $\left(1.0 \mathrm{ml}\right.$ of $1.2 \% \mathrm{NH}_{4} \mathrm{NO}_{3}$ added to $100 \mathrm{ml}$ of a solution containing $5.0 \mathrm{gm}$ of anhydrous $\mathrm{Na}_{2} \mathrm{CO}_{3}, 300 \mathrm{ml}$ of $95 \%$ ethanol, brought to 1.01 with distilled water), followed by immersion in developing solution $\left(1.0 \mathrm{ml}\right.$ of $1.2 \% \mathrm{NH}_{4} \mathrm{NO}_{3}$ and $100 \mathrm{ml}$ of a solution consisting of $0.5 \mathrm{gm}$ of citric acid in $15.0 \mathrm{ml}$ of $37 \%$ formalin, $100 \mathrm{ml}$ of $95 \%$ ethanol, $700 \mathrm{ml}$ of water brought to $\mathrm{pH} 5.8-6.1$ with $9 \% \mathrm{NaOH}$, and finally brought to 1.01 with water). Sections were kept in developing solution for $\geq 1 \mathrm{~min}$. Sections were then mounted on subbed slides, air-dried, and immersed in $0.5 \%$ acetic acid three times for $10 \mathrm{~min}$ each. Sections were then dehydrated through alcohols, cleared in xylene, and coverslipped under Permount.

In situ end-labeling. For in situ end-labeling (ISEL) of free $3^{\prime}$ ends in fragmented nuclear genomic DNA, the rats were perfused with $0.9 \%$ saline for $3 \mathrm{~min}$ and $4 \% \mathrm{PF}$ in $0.1 \mathrm{M} \mathrm{PB}$ for $5 \mathrm{~min}$ only. Brains were post-fixed overnight and then, after cryoprotection and rapid freezing, were serially sectioned at $14 \mu \mathrm{m}$ through the SN. For ISEL, slides were thawed, briefly immersion-fixed in $4 \%$ paraformaldehyde, and rinsed in PBS. Sections were treated with $0.1 \%$ pepsin in $0.01 \mathrm{~N} \mathrm{HCl}$ for $60 \mathrm{~min}$. After another rinse, sections were incubated with terminal deoxynucleotidyl transferase in the presence of digoxigenin-dUTP (ApopTag, Oncor) following the supplier's instructions. Sections were rinsed and then incubated with peroxidase-labeled anti-digoxigenin antibodies (ApopTag). After a rinse, sections were incubated with diaminobenzidine in the presence of $\mathrm{H}_{2} \mathrm{O}_{2}$. Sections were then counterstained with thionin to define intensely basophilic chromatin clumps, characteristic of apoptosis. It is important to note that identification of the morphological features of apoptosis at a cellular level is critical to the interpretation of peroxidaselabeled free $3^{\prime}$ ends, because false-positive staining occurs in cells undergoing necrotic death (Grasl-Kraupp et al., 1995). Positive controls were neonatal tissue sections of the $\mathrm{SN}$ in which apoptosis had been induced by intrastriatal quinolinic acid injection, as reported previously (Macaya et al., 1994).

Quantitative morphological analysis. The prevalence of apoptotic profiles was determined on both TH/Nissl- and silver-stained sections. TH/ Nissl-stained sections were classified according to location in PaxinosWatson planes 2.7, 3.2, 3.7, and 4.2, and all planes were represented from 46 of the 50 brains processed for the analysis of animals lesioned at PND7; in the remaining four brains, 2.7 sections were not available, so they were not analyzed. A mean of $13.5 \pm 0.5$ (SEM) sections per brain were examined. On each section, either the experimental (ipsilateral to 6-OHDA lesion) or control (contralateral to lesion) SN or both were examined at $600 \times$ magnification by scanning from medial to lateral in strictly consecutive and complete dorsal-to-ventral sweeps guided by an eyepiece reticle grid. TH-positive apoptotic profiles were counted if more than half of their area was included within the grid. Counts from sections within each plane were averaged to give a mean count per plane; the values for each of the four planes were added to provide an index of the number of profiles per SN. We did not attempt to determine the proportion of the SN sampled by our sections, and hence the absolute values of profiles per SN. At a cellular level, profiles were counted only if they met the following criteria: (1) one or more intensely basophilic, rounded, and distinct chromatin clumps defined by Nissl stain within a discernible cell nucleus; (2) the presence of a surrounding brown peroxidase-labeled cytoplasm, indicative of the TH-positive phenotype; and (3) the presence of features (1) and (2) within the same planes of focus. Our insistence on the same plane of focus ensures that nuclear apoptotic profiles are not simply superimposed on TH-positive cytoplasm, and it ensures that the top of the apoptotic profile is within the section. This three-dimensional assessment of the profiles is a stereological approach (Gundersen, 1986). For this reason, our data are presented as raw counts, representing tops of profiles within the section. In our assessment of these profiles, the bottom of the nuclear chromatin masses was universally also within the section. This is in keeping with the experience of Clarke and Oppenheim (1995), who have noted that apoptotic profiles are rarely split by the microtome knife, when examined in strictly adjacent sections, even in paraffin-embedded sections cut at $8-12 \mu \mathrm{m}$. We have made similar observations in $20 \mu \mathrm{m}$ frozen sections obtained from SN. Therefore, on the basis of the stereological assessment and these empirical observations, we have not corrected for the theoretical possibility of doublecounting error.

Silver-stained sections were examined at $600 \times$ magnification, as described above. At a cellular level, profiles were counted if they contained one or more intensely argyrophilic, rounded, and distinct chromatin clumps surrounded by a cellular profile. Bare chromatin clumps were not counted, because more than one clump can disperse from a single disintegrating cell. Early in the course of our analysis, we noticed that silver-stained apoptotic profiles appeared to be either of two types: one type was small $(<5 \mu \mathrm{m})$ and contained only one or two chromatin clumps; the other was larger ( $\geq 5 \mu \mathrm{m})$, often contained two or more clumps, and sometimes was surrounded by a neuronal cytoplasmic configuration. For this reason, we counted silver-stained apoptotic profiles as either small $(<5 \mu \mathrm{m})$ or large $(\geq 5 \mu \mathrm{m})$. As for the TH/Nissl-stained material, apoptotic profiles were assessed by focusing up and down to ensure that chromatin clumps were in the same plane as a surrounding cellular profile. Therefore, on the basis of considerations outlined above, data are presented as uncorrected counts.

Quantitative analysis of the extent of TH-positive fiber staining within 
the striatum was based on a segmented field approach (Mize et al., 1988; Burke et al., 1990). This analysis was restricted to four animals lesioned at PND14 and six animals lesioned at PND28. Two or more sections from Paxinos-Watson planes 9.2 and 9.7 were assessed $(5 \pm 0.2$ sections/ animal). Each striatal section was digitized, and the total striatal crosssectional area on the experimental and control side was measured. The maximal optical density value for the TH staining was determined on the control side. "Specific TH" immunostaining was then defined as all staining with an optical density between 50 and $100 \%$ of the maximum. The percentage of the striatal area occupied by this staining was then determined on both sides. For each section, the value for area on the experimental side was normalized by the value on the control side. Data from all sections were averaged to obtain a value for each animal.

The effect of 6-OHDA lesion on the cross-sectional area of the striatum was examined in four PND7 animals at $7 \mathrm{~d}$ postlesion. Nissl-stained coronal sections from planes 9.2 and 9.7 (Paxinos and Watson, 1982) (three to six per animal) were digitized, and the area of the striatum was determined on the control and experimental sides. Area values from each section on each side were averaged to determine values for each animal.

\section{RESULTS}

Neonatal intrastriatal injection of 6-OHDA induced the degeneration of numerous fibers within the striatum, as demonstrated by silver stain (Fig. $1 A$ ) and an extensive loss of TH-positive fibers (Fig. $1 B$ ). As anticipated, there was no apparent injury to intrinsic striatal neurons, indicated by the absence of impregnated neurons on silver stain, and by the absence of any focal loss of neurons on Nissl stain (Fig. 1C). Although there was no apparent focus of neuron loss within the striatum, there was a small $(20 \%)$ but significant decrease in the mean cross-sectional area of the striatum in rats studied at $7 \mathrm{~d}$ after a PND7 lesion (experimental $=6.4$ $\mathrm{mm}^{2}$; control $\left.=8.2 \mathrm{~mm}^{2} ; n=4 ; p<0.01\right)$. In the absence of focal neuron loss, this decrease in area may be related, in part, to the extensive loss of TH-positive neuropil.

Postnatal destruction of striatal dopaminergic terminals resulted in an induction of apoptotic cell death within dopaminergic neurons of the SN. These neurons could be identified by a TH-immunostained cytoplasm encompassing a clear nuclear region that contained one or more intensely basophilic, rounded, sharply delineated chromatin clumps, as is characteristic of apoptosis identified by Nissl counterstain (Fig. 2) (Janec and Burke, 1993; Macaya et al., 1994; Clarke and Oppenheim, 1995). The apoptotic nature of this cell death was confirmed by the demonstration of intensely argyrophilic, rounded, and discrete chromatin clumps on silver stain in neurons of the $\mathrm{SN}$, in sections adjacent to those demonstrating Nissl-positive chromatin clumps (Fig. 3A). This appearance of chromatin clumps on silver stain has been shown to correspond to electron-dense chromatin clumps, characteristic of apoptosis, on ultrastructural study of apoptotic dopaminergic neurons of the SN (Macaya et al., 1994). The apoptotic nature of this morphological pattern of cell death was confirmed further by histochemical demonstration of abundant free $3^{\prime}$ ends, in the nucleus of the nigral cells in association with basophilic chromatin clumps, again demonstrated by Nissl counterstain (Fig. $3 B$ ). As noted previously, we observed two types of silver-stained apoptotic profiles. The type exemplified in Figure $3 A$ was $>5 \mu \mathrm{m}$ in diameter and contained multiple chromatin clumps. The other type, illustrated in Figure $3 C$, was smaller $(<5 \mu \mathrm{m})$ and typically contained only one or two chromatin clumps. Both types showed positive end-labeling.

Quantitative analysis of the number of TH-positive apoptotic profiles at varying time points after injection of 6-OHDA on PND7 showed that an induction of cell death could be identified first on PLD3; it persisted at high levels through PLD7 and had largely abated by day 10 (Fig. 4). The number of TH-positive
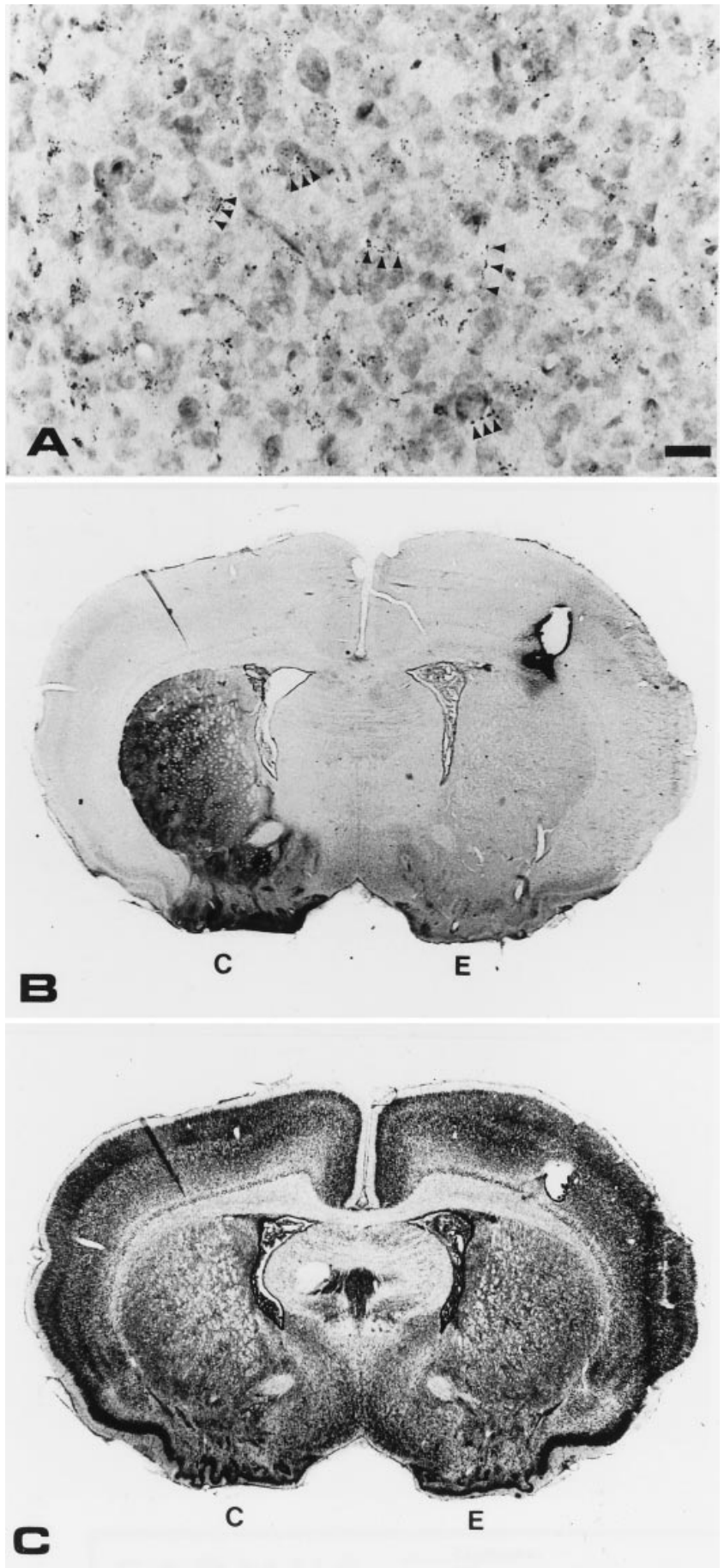

Figure 1. 6-OHDA lesion of striatal dopaminergic terminals. $A$, Photomicrograph of silver-impregnated, degenerating fibers in the striatum after 6-OHDA injection. This animal was injected on PND7 and killed on PLD5. Degenerating fibers are demonstrated by punctate silver deposits, sometimes arranged in linear arrays (arrowheads). Note that there are no degenerating, silver-impregnated neurons. Scale bar, $20 \mu \mathrm{m}$. B, Coronal section of forebrain demonstrating loss of striatal $\mathrm{TH}$ immunostaining on the experimental $(E)$ side after injection of 6-OHDA on PND7. This section is taken from the same animal shown in $A$. There is a complete loss of $\mathrm{TH}$ positivity within the experimental striatum. The oval defect above the striatum on the experimental side was made by the injection cannula. On the control $(C)$ side, an increasing medial-to-lateral gradient for the density of TH staining is apparent. $C$, Coronal section adjacent to the one shown in $B$, stained for Nissl substance. There is a normal configuration of the striatum on the $E$ side, with preservation at cross-sectional area, and there is no evidence for focal neuron loss. 


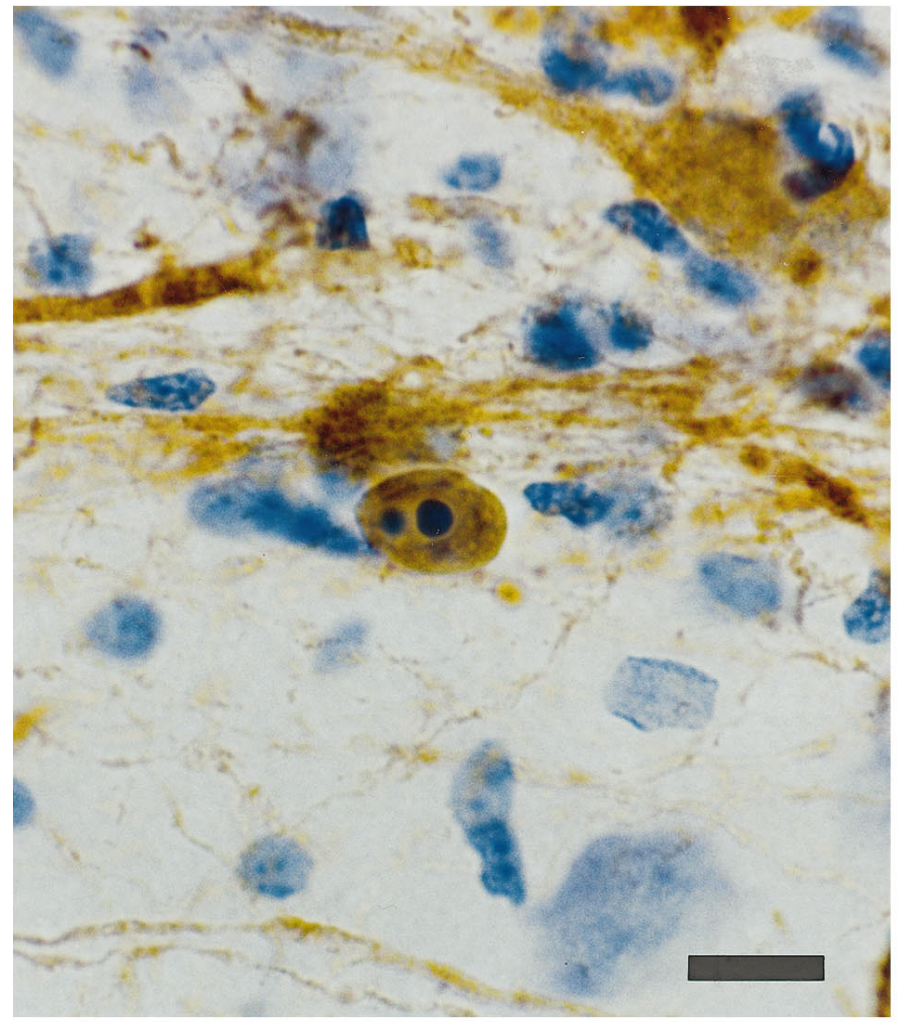

Figure 2. An example of a peroxidase-stained TH-positive neuron in the SNpc with apoptotic nuclear chromatin clumps demonstrated by Nissl counterstain. Scale bar, $10 \mu \mathrm{m}$.
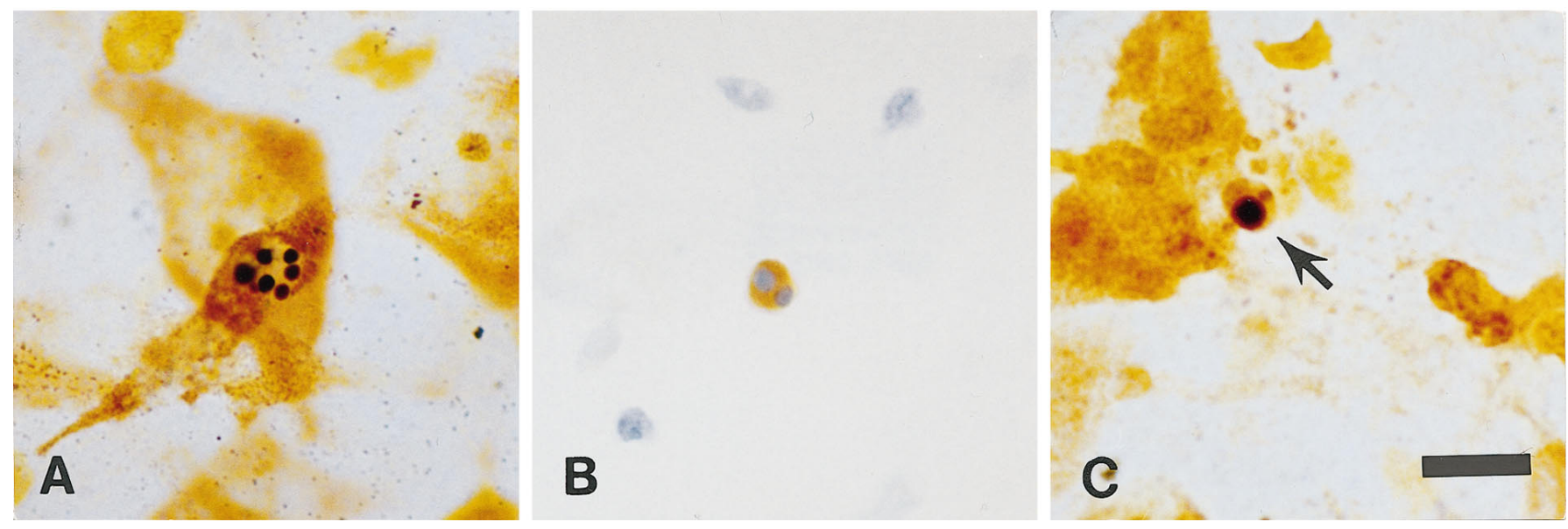

Figure 3. Apoptotic features of cell death in SNpc after striatal 6-OHDA injection. $A$, Silver impregnation demonstrating multiple, rounded, intensely argyrophilic chromatin clumps, characteristic of apoptosis, within the nucleus of a neuron in the SNpc at $5 \mathrm{~d}$ after intrastriatal injection of 6-OHDA on PND14. $B$, ISEL indicated by brown peroxidase reaction product in an apoptotic nucleus, with basophilic chromatic clumps defined by Nissl counterstain. This profile was identified in the SNpc at $6 \mathrm{~d}$ after 6-OHDA on PND7. C, An example of the smaller type of apoptotic profile identified by silver stain. These profiles were $<5 \mu \mathrm{m}$ and contained only one or two chromatin clumps. Scale bar, $10 \mu \mathrm{m}$.

apoptotic neurons reached an apparent maximum by PLD4. Although there was a tendency for values to be higher at PLD6 and -7 , these values were not significantly higher than those observed on days 4 and 5 (ANOVA). The time course and apoptotic nature of the cell death in SN after 6-OHDA injection at PND7 was confirmed by quantitative analysis of large $(>5 \mu \mathrm{m})$ apoptotic profiles in SNpc in silver-stained sections, which showed a maximal induction of cell death from PLD4-7 (Fig. 4B). To examine whether there was any rostrocaudal gradient for the time course of induced cell death after 6-OHDA injection, we classified sections according to rostrocaudal location [using Paxinos and
Watson (1982) reference planes 4.2, 3.7, 3.2, 2.7]) and quantified the number of TH-positive apoptotic profiles (Fig. $4 C$ ). We found that the time course was essentially the same among these planes.

To determine whether induction of apoptotic cell death in the dopaminergic neurons of the $\mathrm{SN}$ is associated with induced cell death of nondopaminergic neurons of the SNpr, we counted the number of TH-negative Nissl-defined apoptotic profiles in the SNpr on the sixth day postlesion, a time when induced cell death in $\mathrm{SNpc}$ had been ongoing for $2 \mathrm{~d}$. This analysis was confined to the anterior-most planes (Paxinos-Watson 4.2 and 3.7), where there are very few $\mathrm{TH}$-positive neurons in SNpr. At that time 

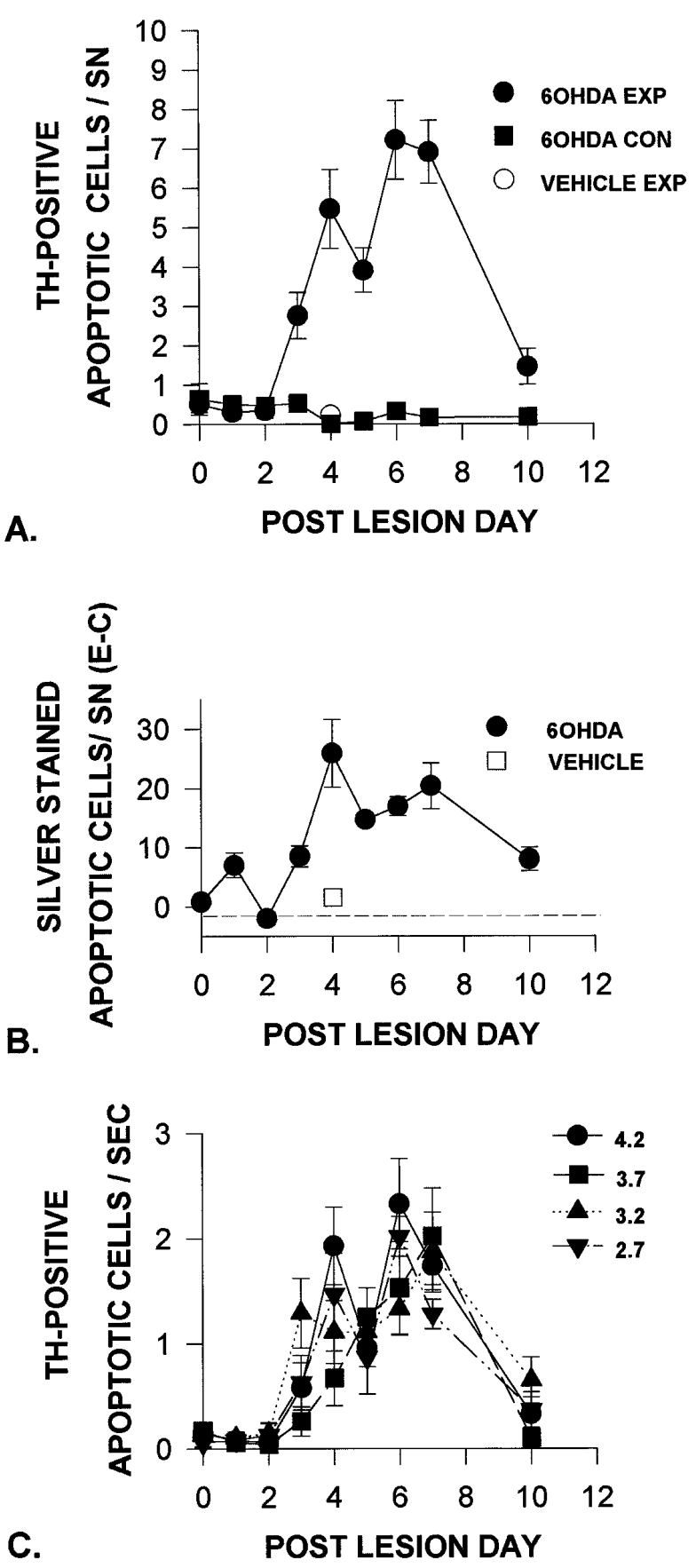

Figure 4. A, Time course for induction of apoptosis in dopaminergic neurons within the SN after injection of 6-OHDA on PND7. The number of TH-positive apoptotic profiles was determined as described in Materials and Methods on the side of the 6-OHDA injection (6OHDA EXP) $(\bullet)$, on the contralateral control side $(6 O H D A C O N)(\mathbf{\square})$, and on the ipsilateral side in vehicle-injected controls $(\bigcirc)$. An augmented level of cell death was first noted on PLD3 and abated by day 10. $B$, Time course for induction of apoptosis in $\mathrm{SN}$, assessed by counts of the total number of silver-stained apoptotic profiles. Silver staining is more sensitive to the presence of apoptotic profiles and includes cells of all phenotypes, so higher counts are obtained; note the difference in the ordinate scale, as compared with $A$. Because silver staining more readily reflects the changing level of natural cell death during this postnatal period (Janec and Burke, 1993), we have for ease of comparison to $A$ expressed counts as the total number of apoptotic profiles on the experimental side minus the level of natural cell death on the control side. $C$, An analysis of induced apoptotic cell death in TH-positive neurons according to rostrocaudal location shows that the time course is the same in Paxinos-Watson (Paxinos and Watson, 1982) planes $4.2,3.7,3.2$, and 2.7. point, there was no significant difference in Nissl-positive apoptotic profiles in SNpr on the 6-OHDA-lesioned side (3.1 \pm 0.8 cells per $\mathrm{SNpr}$ ) in comparison to the contralateral control side $(3.8 \pm 1.0$ cells $)$. Thus, induction of apoptotic cell death in the dopaminergic neurons of the SN did not appear to be associated with a similar induction in the SNpr.

The developmental dependence of the ability of intrastriatal 6-OHDA to induce apoptotic cell death in the SNpc was investigated in animals at PND7, -14, -21, and -28. For this analysis, all animals were examined at PLD5, and apoptotic profiles were identified by silver staining. Profiles were differentiated into small $(<5 \mu \mathrm{m})$ and large $(\geq 5 \mu \mathrm{m})$. As shown in Figure $5 A$, there was a clear developmental difference in the ability of 6-OHDA to induce apoptotic cell death in SNpc. At PND7 and -14, there was a four- and threefold induction of apoptotic cell death, respectively, assessed by counting all apoptotic profiles in SNpc $(p<0.0001$, ANOVA), whereas at PND21 and -28, there was no significant effect. Analysis also revealed an effect of developmental age on the number of apoptotic profiles on the control (unlesioned) side: the number of profiles at PND14 was significantly greater than the number at PND28 $(p<0.05$, Bonferroni test). No other differences among control conditions were significant. This observation agrees with our previous finding that there is a peak in developmental cell death in SNpc at PND14 and that it diminishes by PND28 (Janec and Burke, 1993). When the analysis is restricted to small apoptotic profiles (Fig. $5 B$ ), there is no apparent inductive effect of the lesion. Within this category of profile only the effect of developmental age is noted; the number of profiles at both PND14 and -21 were significantly greater than at PND28 ( $p<0.05$, Bonferroni test). The ability of intrastriatal 6-OHDA to induce apoptotic cell death is seen most clearly at early developmental ages in the analysis of large apoptotic profiles, in which there is a highly significant 10 - and 20-fold induction at PND7 and -14 , respectively. Although greater numbers of large apoptotic profiles were also observed on the experimental side at PND21 and -28 , these differences did not quite achieve significance. In this analysis, there was no significant difference in numbers of large profiles on the control side among the different developmental ages. We conclude that there is developmental dependence of induced death in SNpc and that the large apoptotic profiles are likely to derive from the dopaminergic neuron phenotype. This conclusion is supported by finding a similar pattern of induction of TH-positive apoptotic profiles in PND7, -14, and -28 (Fig. 6). The cell phenotype from which the small apoptotic profiles arise is unknown, and the prevalence of these profiles is not affected by the 6-OHDA striatal lesion.

We considered the possibility that the developmental difference in ability to induce apoptotic cell death in SNpc may be related to a difference in the ability of intrastriatal 6-OHDA to induce destruction of striatal dopaminergic fibers. To examine this issue, we performed a segmented field analysis (Burke et al., 1990) of the extent of striatal TH-positive fiber innervation on the lesioned side in comparison to control for animals at PLD14 and -28. This analysis showed that the extent of fiber loss was as great at PND28 (TH-stained area $49.7 \% \pm 7.4$ that of contralateral control; $n=6$ ) as at PND14 (TH-stained area $66.0 \% \pm 12.1 ; n=4$ ). Therefore, the difference in the level of induced apoptotic cell death between these two ages cannot be attributed to differences in the extent of the 6-OHDA-induced lesion.

We also considered the possibility that the apparent lack of induction of apoptotic death at the later PNDs may be attributable to a shift in the time course. In the foregoing experiments, 


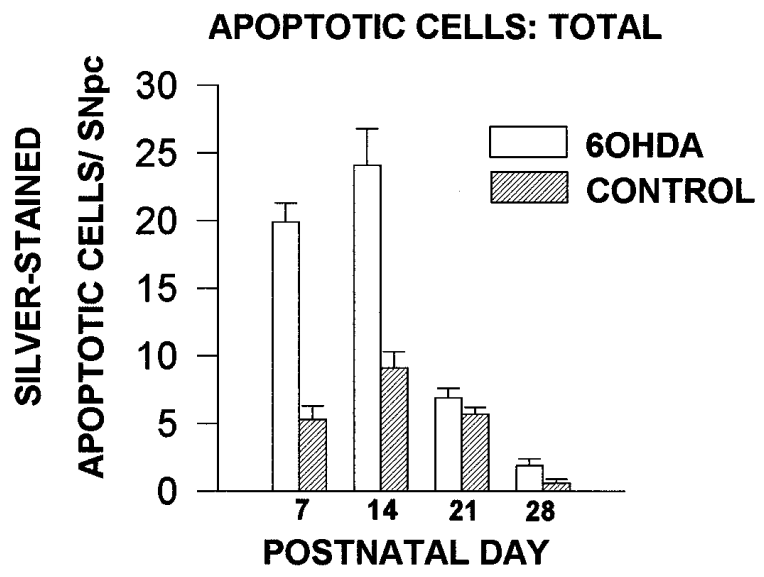

A.

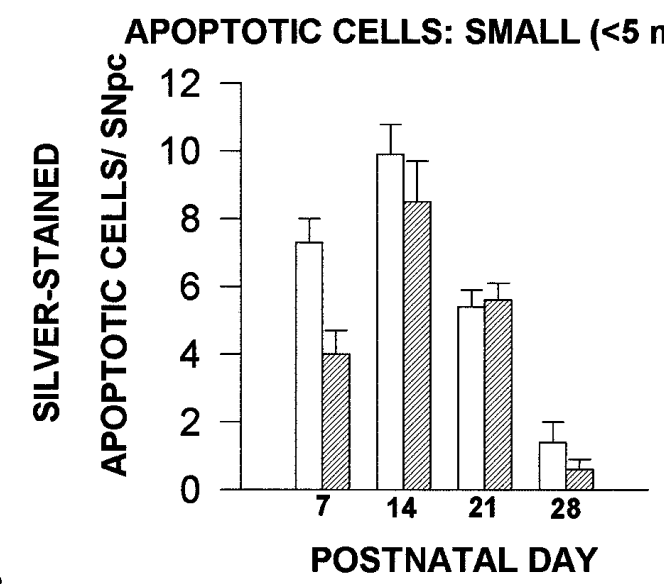

B.

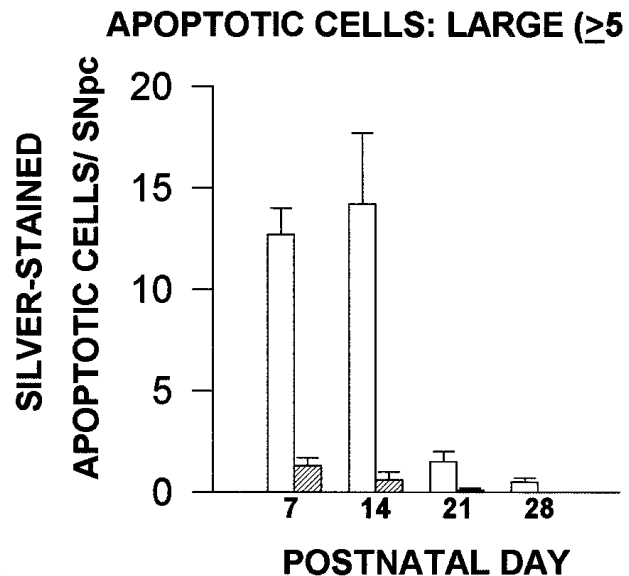

C.

Figure 5. Developmental dependence of induction of apoptotic cell death in SN by striatal 6-OHDA lesion. Animals were lesioned on the indicated postnatal day and killed $5 \mathrm{~d}$ later. For PND7, the data are derived from the same group of animals $(n=6)$ depicted in Figure 4 at PLD5. An additional 20 animals were studied for the subsequent PNDs. $A$, Counts of the total number of silver-stained apoptotic profiles for the 6-OHDA and contralateral control sides. There was an induction of cell death on PND7 and -14. B, There was no apparent induction of cell death assessed by counting small apoptotic profiles. $C$, Large profiles show a marked induction of death at PND7 and -14. Although there is an apparent trend for an effect at PND21 and -28, ANOVA does not achieve significance.

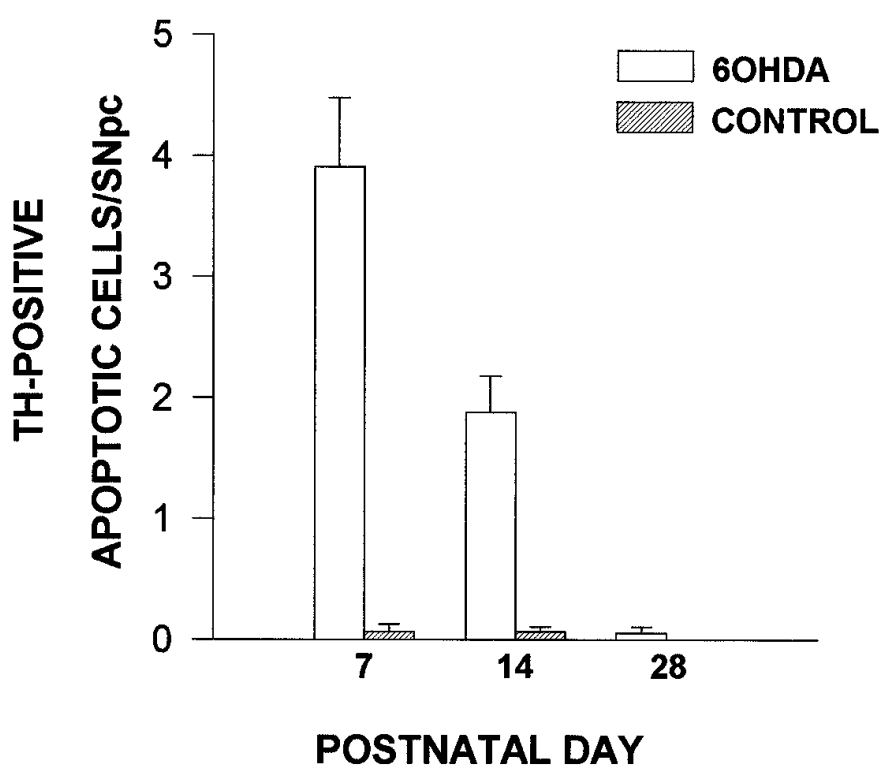

Figure 6. An analysis of TH-positive apoptotic profiles indicates that a developmental dependence for induction of apoptotic cell death in SN is also observed in phenotypically defined dopaminergic neurons.

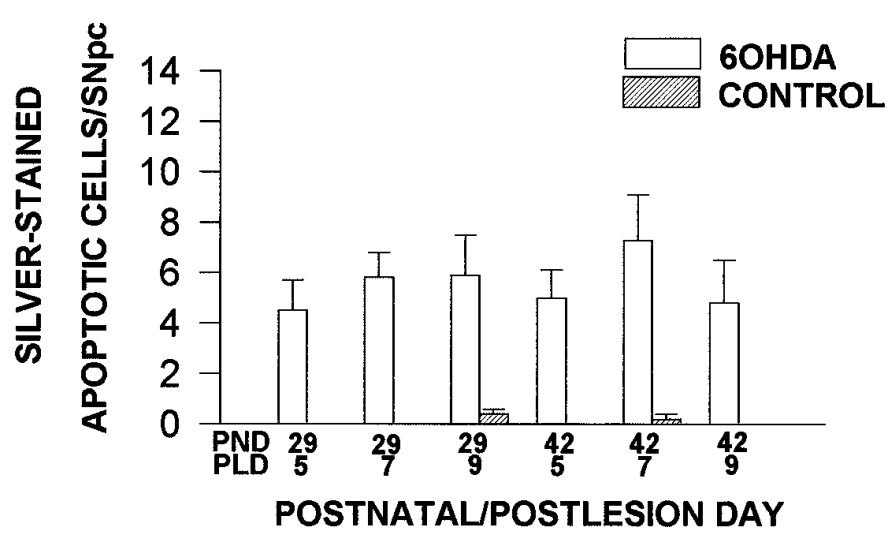

Figure 7. Time course of induced apoptotic cell death in SNpc at later postnatal ages. Only data for large $(\geq 5 \mu \mathrm{m})$, silver-stained profiles are presented. Thirty animals underwent 6-OHDA lesion at the indicated PNDs and were killed after 5, 7, or $9 \mathrm{~d}$. In this analysis restricted to these later postnatal ages, there is a clearly significant induction of cell death $(p<0.0001)$ at all PNDs and postlesion times; however, there was no significant effect of postlesion interval.

animals were killed at PLD5, which is within the period of maximal induction of cell death at PND7 (Fig. 4). It is possible that at later postnatal ages there is a shift in the time course toward a later occurrence. To examine this issue, animals at PND29 and -42 were subjected to 6-OHDA lesion and killed at PLD5, -7, and -9. Quantitative analysis of the number of large $(>5 \mu \mathrm{m})$ silverstained apoptotic profiles in SNpc showed that the level of cell death did not increase at later postlesion intervals, at either PND29 or PND42 (Fig. 7). In this experiment, in the absence of the large effects observed previously at PND7 and -14, and in the absence of the greater variability observed in the complete developmental study, ANOVA reveals a very clear induction of cell death in SNpc at all postlesion intervals for PND29 and -42 $(p<$ $0.0001)$. Thus, what was apparent only as a trend at later developmental ages (PND21 and -28) in the earlier, complete developmental study (Fig. $5 C$ ) is revealed here as a significant effect at 

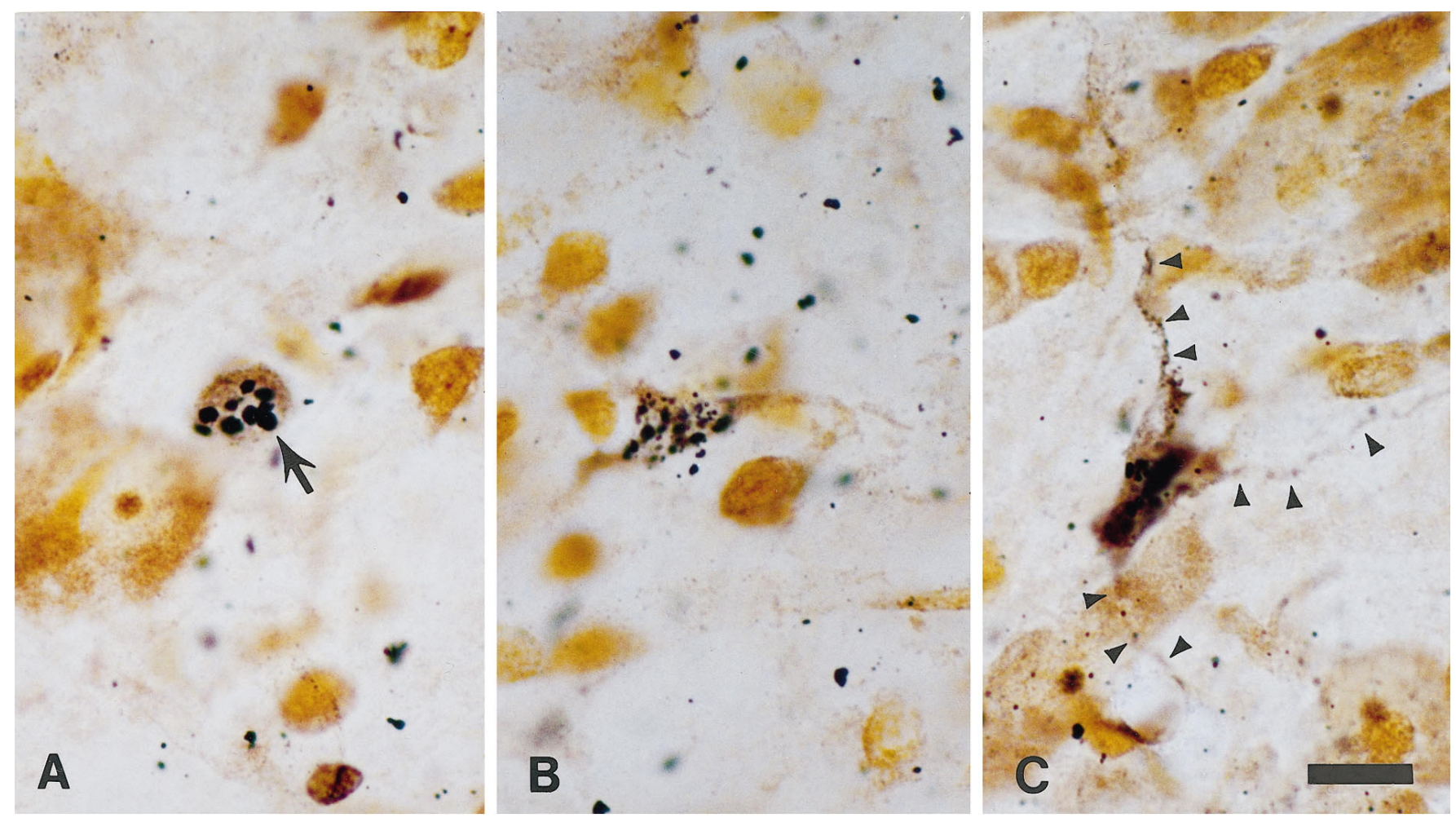

Figure 8. The morphology of cell death at PND42 is not uniformly apoptotic, as shown in these silver-stained sections from animals $9 \mathrm{~d}$ after lesion. Although apoptosis does occur, as shown in $A$ (a typical argyrophilic chromatin clump is indicated by an arrow), nonapoptotic morphology is also observed in the same section, as shown in $B$. This cell shows pleiomorphic, irregular silver deposits in the nucleus. A second example of nonapoptotic morphology is shown in $C$, in which there is heavy silver impregnation throughout the nucleus and cytoplasm. The cytoplasmic impregnation extends into dendrites, marked by arrowheads. Scale bar, $10 \mu \mathrm{m}$.

PND29 and -42. Nevertheless, the magnitude of this effect is less than what was observed previously at PND7 and -14 , and this difference cannot be attributed to a difference in the time course of induced death at later postnatal ages. In this late developmental study, we made the additional observation that the morphological pattern of induced cell death was not uniformly apoptotic, as it was at earlier ages. Some of the silver-impregnated cells in PND42, PLD9 animals showed a diffuse impregnation of the nucleus and cytoplasm rather than classic apoptotic chromatin clumps. Such nonapoptotic profiles were often interspersed in the same section with apoptotic profiles (Fig. 8). These nonapoptotic profiles were not included in our counts of apoptotic profiles, as presented in Figure 7. They were not present at PND29, however, and very few were present at PND42, so they would not account for the difference in the level of induced cell death observed in later as compared with earlier developmental ages.

\section{DISCUSSION}

We have identified apoptosis within developing dopaminergic neurons after destruction of their terminals with 6-OHDA using both morphological and histochemical criteria. The demonstration of intensely basophilic, rounded, and well demarcated chromatin clumps using Nissl stains has been considered by many investigators to be highly suggestive of apoptosis at the light microscope level (Clarke and Oppenheim, 1995), and in many instances this has been confirmed by ultrastructure (Cunningham, 1982; Williams and Rakic, 1988; Ferrer et al., 1990; Sloviter et al., 1993; Macaya et al., 1994). The apoptotic nature of such clumps can be confirmed by silver impregnation techniques, which demonstrate intensely argyrophilic, rounded intranuclear clumps
(Yamamoto et al., 1986; Janec and Burke, 1993; Macaya et al., 1994; Mitchell et al., 1994), and which have also been demonstrated by ultrastructure (Macaya et al., 1994) to conform to classic descriptions of apoptosis (Kerr et al., 1995). In addition, we have shown that apoptosis occurs by demonstrating a high concentration of free $3^{\prime}$ ends within the nucleus in the presence of basophilic chromatin clumps, visualized with Nissl counterstain. Although the ISEL technique can produce false-positive labeling of free $3^{\prime}$ ends generated by a nonapoptotic process (GraslKraupp et al., 1995), it is reliable if, in addition to the histochemical reaction, the morphological features of apoptosis are identified (Oo et al., 1995). The induction of apoptosis specifically within dopaminergic neurons has been demonstrated by $\mathrm{TH}$ immunostaining within the cytoplasm in conjunction with Nissl counterstain of nuclear chromatin clumps.

The developmental dependence of intrastriatal 6-OHDA to induce apoptotic cell death was observed most readily in a population of silver-stained apoptotic profiles with diameters $>5 \mu \mathrm{m}$. Within this group, at PND7 and -14, the number of profiles on the side of experimental injury was increased by an order of magnitude over the numbers observed on the contralateral control side and on either side at PND21 and -28. Within the group of profiles with diameters $<5 \mu \mathrm{m}$, there was no apparent inductive effect of the lesion at any postnatal age. One possible interpretation of these results is that the experimental injury simply led to an increase in the size of the apoptotic profiles at early postnatal ages, and the apparent increase in total and large profiles at these ages is attributable to double-counting error (Abercrombie, 1946). If that were true, however, then the number of small 

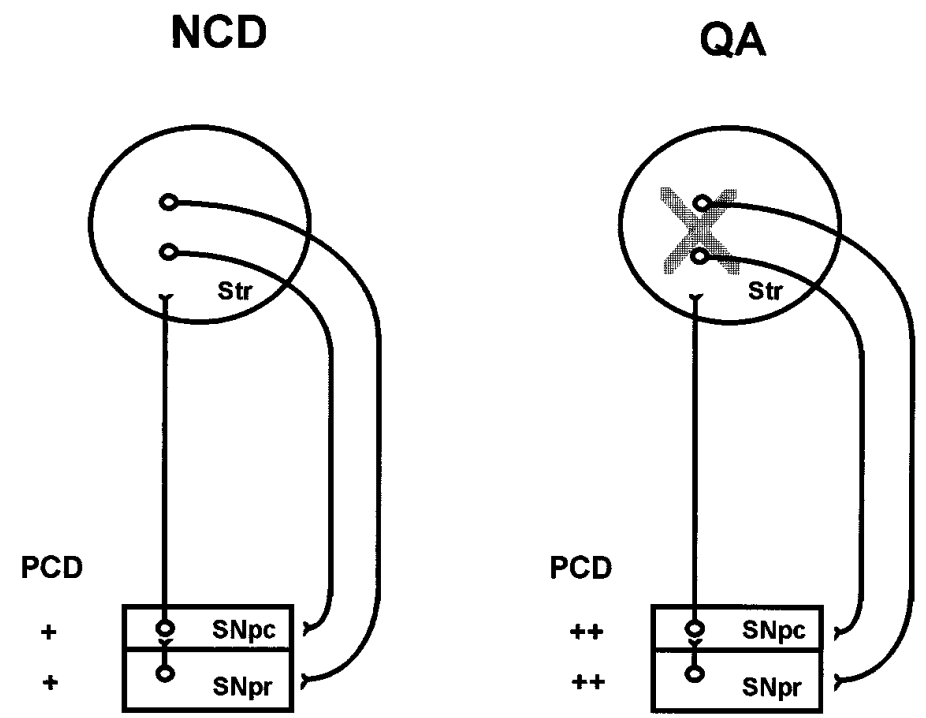

\section{OHDA}

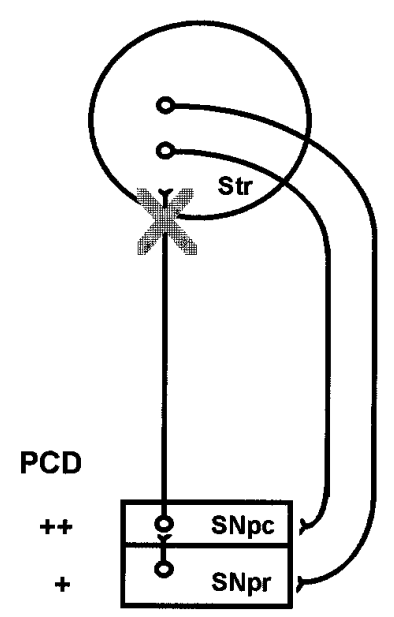

Figure 9. Summary of observations related to natural and induced cell death in SN. The relevant anatomy has been highly simplified to show only the nigrostriatal dopaminergic projection, the striatonigral projections to SNpc and SNpr (Gerfen et al., 1987), and interactions between GABAergic neurons of the SNpr and dopaminergic neurons of the SNpc (Tepper et al., 1995). Natural cell death (NCD) with the morphology of apoptosis occurs in both SNpc and SNpr. After quinolinate $(Q A)$ lesion of intrinsic striatal neurons, which spares dopaminergic terminals, there is an induction of cell death, with apoptotic morphology in SNpc and SNpr (Macaya et al., 1994). Destruction of dopaminergic terminals alone with 6-OHDA induces cell death only in SNpc.

profiles at PND7 and -14 would decrease, but they did not. In addition, the finding of a greater induction of apoptotic cell death at PND7 and -14 in relation to PND28 was confirmed for neurons with the dopaminergic phenotype, using a stereological counting approach (Figs. 4A, 6). Therefore, we conclude that the large apoptotic profiles are derived, at least in part, from the dopaminergic phenotype, and the smaller profiles derive from another, unknown cellular phenotype. This latter phenotype demonstrates developmental regulation (Fig. $5 B$ ), but it is not induced to undergo apoptosis after destruction of dopaminergic terminals. The developmental dependence of the inductive effect was not attributable to the ability of 6-OHDA to produce a more extensive lesion at younger ages. Analysis of the loss of TH-immunostained striatal fibers showed comparable effects at PND14 and -28. Nor could the apparent developmental dependence be attributed to a delay in the time course of induced death in the older animals.

These results are compatible with our hypothesis that during development, dopaminergic neurons of the $\mathrm{SN}$ are dependent on their target, the striatum, for viability. Destruction of the terminals of these neurons with 6-OHDA would be expected to eliminate their ability to interact with their target, either by the uptake of diffusible trophic molecules or by cell-to-cell contact. Our results indicate that the developing dopaminergic neurons are dependent on the striatal target on PND7 through PND14. This observation is similar to findings in other developmental paradigms in which the period of dependence on the target for viability is restricted to a critical window of time. For example, there is evidence that rat sensory neurons lose their nerve growth factor (NGF) dependence over a 2 week period after birth (Lindsay, 1993). The possibility that the developing dopaminergic system is target-dependent on PND7 through PND14 is supported by our recent observation that induced apoptotic cell death in $\mathrm{SN}$ after early excitotoxic striatal injury shows a similar developmental dependence; there is a marked decrease in induced cell death after PND14 (Kelly and Burke, 1996).

Another possible interpretation of our results is that the induc- tion of apoptotic cell death in $\mathrm{SN}$ is unrelated to target dependence but reflects a direct toxic effect of the 6-OHDA injection. 6-OHDA can induce apoptosis in vitro (Walkinshaw and Waters, 1994). In adult animals, injection of 6-OHDA into striatum results in the death of dopaminergic neurons (Sauer and Oertel, 1994). In adult animals, however, direct injection of 6-OHDA into SN (Jeon et al., 1995) or injection into striatum (Ichitani et al., 1991, 1994) does not appear to induce apoptotic cell death. Nevertheless, it remains possible that immature dopaminergic neurons respond differently to the toxin than mature neurons do, and they die with an apoptotic morphology. Such a possibility, however, although it may account for the appearance of apoptosis at early developmental ages, would not account for the increased magnitude of cell death at earlier ages. On the contrary, given that the toxic effect of 6-OHDA is dependent on the dopamine reuptake site (Kostrzewa and Jacobowitz, 1974), and that these sites are relatively less numerous in early development (Coyle, 1977), one would anticipate that smaller numbers of neurons would be injured by a direct toxic effect in immature animals. In addition, if the induction of apoptosis in young animals were to be strictly attributable to a toxic effect, then it would be only coincidental that both the induction of apoptosis by 6-OHDA and the induction by excitotoxic striatal injury have the same developmental time course, with a marked decrease of induced cell death after PND14. It seems most parsimonious, therefore, to attribute the ability of 6-OHDA to induce cell death in immature animals to interference with target-derived support. This hypothesis is further testable by administration of exogenous putative trophic factors to ascertain whether they might inhibit 6-OHDA-induced apoptotic cell death, analogous to classic studies of NGF support of sympathetic neurons after destruction of their terminals by 6-OHDA (Levi-Montalcini et al., 1975).

The observations made in the present investigation and in earlier studies of natural and induced cell death in $\mathrm{SNpc}$ are summarized in Figure 9. During normal development, natural cell death occurs with the morphology of apoptosis within both the 
SNpc (Janec and Burke, 1993) and the SNpr (Szeto et al., 1994). The magnitude of this death event is increased in both structures after early (PND7) excitotoxic quinolinic acid (QA) injury to the striatum (Macaya et al., 1994). This lesion spares the terminals of the nigrostriatal dopaminergic projection (Schwarcz et al., 1983; Macaya and Burke, 1992). In both structures, the morphology of the induced death event is apoptotic and indistinguishable from natural cell death. There are multiple possible relationships between the experimental lesion in the striatum and the induced death in SN. For example, although the striatum is a major target of the nigrostriatal projection, the $\mathrm{SNpc}$ also receives important afferent projections from the striatum (Gerfen et al., 1987). The neurons of the SNpr receive a major afferent projection from striatum, but they also have projections to $\mathrm{SNpc}$ as one of their targets (Tepper et al., 1995). In the present study, the terminals of the nigrostriatal dopaminergic projection have been selectively injured, without significant damage to striatal intrinsic neurons. This lesion results in an induced death event in SNpc but not in SNpr. This result would suggest that disruption of target interaction is sufficient to induce cell death in SNpc and that disruption of afferent projections are not necessary to induce death. This result does not exclude the possibility that afferent projections may be capable of regulating cell death in developing SNpc. The absence of induced death in SNpr suggests that augmented death in one of its targets (the SNpc) is not sufficient to augment the natural cell death event. This may be because SNpr has other collateral projections (Tepper et al., 1995). This result would suggest that the induced cell death in SNpr in the QA paradigm was not attributable to augmented cell death in SNpc but rather to loss of striatal afferent projections. In both the QA and 6-OHDA models, the time course of developmental dependence for induced death in SNpc is quite similar, with a sharp decrement after PND14.

The observation of two different morphological patterns of induced cell death at PND42 suggests either that 6-OHDA induces cell death at that age by two different mechanisms or that the same mechanism causes two different morphologies of cell death. In the former instance, it is possible, for example, that the apoptotic cell death is attributable to the loss of target-derived support, whereas the nonapoptotic death is attributable to the direct effect of retrogradely transported toxin (Ichitani et al., 1991, 1994; Sauer and Oertel, 1994). In the latter instance, it is possible that as the animal matures the morphology changes from an apoptotic to a nonapoptotic form, while the death mechanism remains that of programmed cell death. Such a transition has been observed in other studies of natural and induced developmental cell death (Pilar and Landmesser, 1976; Cunningham, 1982). It will be possible to differentiate between these alternate explanations by biochemical and morphological characterization of these different types of cell death.

\section{REFERENCES}

Abercrombie M (1946) Estimation of nuclear populations from microtome sections. Anat Rec 94:239-247.

Barde YA (1989) Trophic factors and neuronal survival. Neuron 2:1525-1534.

Burke RE, Cadet JL, Kent JD, Karanas AL, Jackson Lewis V (1990) An assessment of the validity of densitometric measures of striatal tyrosine hydroxylase-positive fibers: relationship to apomorphine-induced rotations in 6-hydroxydopamine lesioned rats. J Neurosci Methods 35:63-73.

Burke RE, Macaya A, DeVivo D, Kenyon N, Janec EM (1992) Neonatal hypoxic-ischemic or excitotoxic striatal injury results in a decreased adult number of substantia nigra neurons. Neuroscience 50:559-569.
Clarke PGH (1985) Neuronal death in the development of the vertebrate nervous system. Trends Neurosci 345-349.

Clarke PGH, Oppenheim RW (1995) Neuron death in vertebrate development: in vivo methods. In: Methods in cell biology: cell death (Schwartz LM, Osborne BA, eds), pp 277-321. New York: Academic.

Cowan WM, Fawcett JW, O'Leary DDM, Stanfield BB (1984) Regressive events in neurogenesis. Science 225:1258-1265.

Coyle JT (1977) Biochemical aspects of neurotransmission in the developing brain. Int Rev Neurobiol 20:65-102.

Cunningham TJ (1982) Naturally occurring neuron death and its regulation by developing neural pathways. Int Rev Cytol 74:163-186.

Ferrer I, Bernet E, Soriano E, del Rio T, Fonseca M (1990) Naturally occurring cell death in the cerebral cortex of the rat and removal of dead cells by transitory phagocytes. Neuroscience 39:451-458.

Gerfen CR, Herkenham M, Thibault J (1987) The neostriatal mosaic. II. Patch- and matrix-directed mesostriatal dopaminergic and nondopaminergic systems. J Neurosci 7:3915-3934.

Giros B, Jaber M, Jones SR, Wightman RM, Caron MG (1996) Hyperlocomotion and indifference to cocaine and amphetamine in mice lacking the dopamine transporter. Nature 379:606-612.

Grasl-Kraupp B, Ruttkay-Ndicky B, Koudelka H, Bukowska K, Bursch W, Schulte-Hermann R (1995) In situ detection of fragmented DNA (TUNEL assay) fails to discriminate among apoptosis, necrosis, and autolytic cell death: a cautionary note. Hepatology 21:1465-1468.

Gundersen HJG (1986) Stereology of arbitrary particles. J Microsc $143: 3-45$.

Hemmendinger LM, Garber BB, Hoffmann PC, Heller A (1981) Target neuron-specific process formation by embryonic mesencephalic dopamine neurons in vitro. Proc Natl Acad Sci USA 78:1264-1268.

Ichitani Y, Okamura H, Matsumoto Y, Nagatsu I, Ibata Y (1991) Degeneration of the nigral dopamine neurons after 6-hydroxydopamine injection into the rat striatum. Brain Res 549:350-353.

Ichitani Y, Okamura H, Nakahara D, Nagatsu I, Ibata Y (1994) Biochemical and immunocytochemical changes induced by intrastriatal 6-hydroxydopamine injection in the rat nigrostriatal dopamine neuron system: evidence for cell death in the substantia nigra. Exp Neurol 130:269-278.

Janec E, Burke RE (1993) Naturally occurring cell death during postnatal development of the substantia nigra of the rat. Mol Cell Neurosci 4:30-35.

Jeon BS, Jackson-Lewis V, Burke RE (1995) 6-Hydroxydopamine lesion of the rat substantia nigra: time course and morphology of cell death. Neurodegen 4:131-137.

Kelly WJ, Burke RE (1996) Apoptotic neuron death in rat substantia nigra induced by striatal excitotoxic injury is developmentally dependent. Neurosci Lett 220:85-88.

Kerr JF, Wyllie AH, Currie AR (1972) Apoptosis: a basic biological phenomenon with wide-ranging implications in tissue kinetics. $\mathrm{Br} \mathrm{J}$ Cancer 26:239-257.

Kerr JFR, Gobe GC, Winterford CM, Harmon BV (1995) Anatomical methods in cell death. In: Methods in cell biology: cell death (Schwartz LM, Osborne BA, eds), pp 1-27. New York: Academic.

Kostrzewa RM, Jacobowitz DM (1974) Pharmacological actions of 6-hydroxydopamine. Pharmacol Rev 26:199-288.

Levi-Montalcini R, Aloe L, Mugnaini E, Oesch F, Thoenen H (1975) Nerve growth factor induces volume increase and enhances tyrosine hydroxylase synthesis in chemically axotomized sympathetic ganglia of newborn rats. Proc Natl Acad Sci USA 72:595-599.

Linden R (1994) The survival of developing neurons: a review of afferent control. Neuroscience 58:671-682.

Lindsay RM (1993) Brain-derived neurotrophic factor: an NGF-related neurotrophin. In: Neurotrophic factors (Loughlin SE, Fallon JH, eds), pp 257-284. San Diego: Academic.

Macaya A, Burke RE (1992) Effect of striatal lesion with quinolinate on the development of substantia nigra dopaminergic neurons: a quantitative morphological analysis. Dev Neurosci 14:362-368.

Macaya A, Munell F, Gubits RM, Burke RE (1994) Apoptosis in substantia nigra following developmental striatal excitotoxic injury. Proc Natl Acad Sci USA 91:8117-8121.

Mitchell IJ, Lawson S, Moser B, Laidlaw SM, Cooper AJ, Walkinshaw G, Waters CM (1994) Glutamate induced apoptosis results in a loss of striatal neurons in the parkinsonian rat. Neuroscience 63:1-5.

Mize RR, Holdefer RN, Nabors LB (1988) Quantitative immunocytochemistry using an image analyzer. I. Hardware evaluation, image processing, and data analysis. J Neurosci Methods 26:1-24. 
Oo TF, Henchcliffe C, Burke RE (1995) Apoptosis in substantia nigra following developmental hypoxic-ischemic injury. Neuroscience 69:893-901.

Oppenheim RW (1991) Cell death during development of the nervous system. Annu Rev Neurosci 14:453-501.

Paxinos G, Watson C (1982) The rat brain in stereotaxic coordinates. San Diego: Academic.

Pilar G, Landmesser L (1976) Ultrastructural differences during embryonic cell death in normal and peripherally deprived ciliary ganglia J Cell Biol 68:339-356.

Prochiantz A, di Porzio U, Kato A, Berger B, Glowinski J (1979) In vitro maturation of mesencephalic dopaminergic neurons from mouse embryos is enhanced in presence of their striatal target cells. Proc Natl Acad Sci USA 76:5387-5391.

Sauer H, Oertel WH (1994) Progressive degeneration of nigrostriatal dopamine neurons following intrastriatal terminal lesions with 6-hydroxydopamine: a combined retrograde tracing and immunocytochemical study in the rat. Neuroscience 59:401-415.

Schwarcz R, Whetsell WO, Mangano RM (1983) Quinolinic acid: an endogenous metabolite that produces axon-sparing lesions in the rat brain. Science 219:316-318.

Sloviter RS, Dean E, Neubort S (1993) Electron microscopic analysis of adrenalectomy induced hippocampal granule cell degeneration in the rat: apoptosis in the adult central nervous system. J Comp Neurol 330:337-351.

Szeto A, Oo T, Burke RE (1994) Naturally occurring cell death during postnatal development of the substantia nigra pars reticulata of rat. Mov Disord 9:[Suppl 1]:106A.

Tepper JM, Martin LP, Anderson DR (1995) $\mathrm{GABA}_{\mathrm{A}}$ receptormediated inhibition of rat substantia nigra dopaminergic neurons by pars reticulata projection neurons. J Neurosci 15:3092-3103.

Tomozawa Y, Appel SH (1986) Soluble striatal extracts enhance development of mesencephalic dopaminergic neurons in vitro. Brain Res 399:111-124.

Ungerstedt U (1971) Histochemical studies on the effect of intracerebral and intraventricular injections of 6-hydroxydopamine on monoamine neurons in the rat brain. In: 6-Hydroxydopamine and catecholamine neurons (Malmfors T, Thoenen H, eds), pp 101-127. Amsterdam: North Holland.

Walkinshaw G, Waters CM (1994) Neurotoxin induced cell death in neuronal PC12 cells is mediated by induction of apoptosis. Neuroscience 63:975-987.

Wetts R, Herrup K (1983) Direct correlation between Purkinje and granule cell number in the cerebella of lurcher chimeras and wild-type mice. Dev Brain Res 10:41-47.

Williams RW, Rakic P (1988) Elimination of neurons from the rhesus monkey's lateral geniculate nucleus during development. J Comp Neurol 272:424-436.

Yamamoto T, Iwasaki Y, Konno H, Iizuka H (1986) Identification of cells undergoing physiological neuronal death in the neonatal rat brain by the Fink-Heimer method. Brain Res 374:419-424. 